\title{
On the Reconstruction of Finite Lattice Sets from Their X-Rays
}

\author{
Peter Gritzmann* \\ Zentrum Mathematik, Technische Universität München, \\ D-80290 München, Germany \\ gritzman@mathematik.tu-muenchen.de
}

\begin{abstract}
We study various theoretical and algorithmic aspects of inverse problems in discrete tomography that are motivated by demands from material sciences for the reconstruction of crystalline structures from images produced by quantitative high resolution transmission electron microscopy.

In particular, we discuss questions related to the ill-posedness of the problem, determine the computational complexity of the basic underlying tasks and indicate algorithmic approaches in the presence of $\mathbb{N} \mathbb{P}$ hardness.
\end{abstract}

\section{Introduction}

While having a number of interesting applications in and connections to areas like general image processing, graph theory, scheduling, statistical data security, game theoryetc. (see e.g. [16], [28], [10], [21], [14], [15]), the main motivation for the present paper's discussion of the problem of reconstructing finite lattice sets from certain of their marginal sums is the demand from material sciences to reconstruct crystalline structures given through their images under high resolution transmission electron microscopy in a certain limited number of directions. In fact, [29] and [22] show how a quantitative analysis of images from high resolution transmission electron microscopy can be used to determine the number of atoms on atomic columns in certain directions. The goal is to use this technique for quality control in VLSI-technology. In particular, the interfacial topography of a material is vital in the manufacture of silicon chips.

So, in principle, we are given the information how many atoms there are on each line parallel to a certain direction for a certain small number of different directions. To be more precise, let $n \in \mathbb{N}, n \geq 2$, let $F$ be a finite subset of $\mathbb{Z}^{n}$, let $S$ be a line through the origin, and let $\mathcal{A}(S)$ denote the set of all lines of Euclidean $n$-space $\mathbb{E}^{n}$ that are parallel to $S$. Then the (discrete) $X$-ray of $F$ parallel to $S$ is the function $X_{S} F: \mathcal{A}(S) \rightarrow \mathbb{N}_{0}=\mathbb{N} \cup\{0\}$ defined by

$$
X_{S} F(T)=|F \cap T|=\sum_{x \in T} \mathbf{1}_{F}(x),
$$

\footnotetext{
* Supported in part by a Max Planck Research Award and by the German Federal Ministry of Education, Science, Research and Technology Grant 03-GR7TM1.
} 
for $T \in \mathcal{A}(S)$. Of course, in practice only the cases $n=2$ and $n=3$ are relevant.

Figure 1 shows a 3D-sample that has been reconstructed from three of its $\mathrm{X}$-rays by methods outlined in Section 5 .

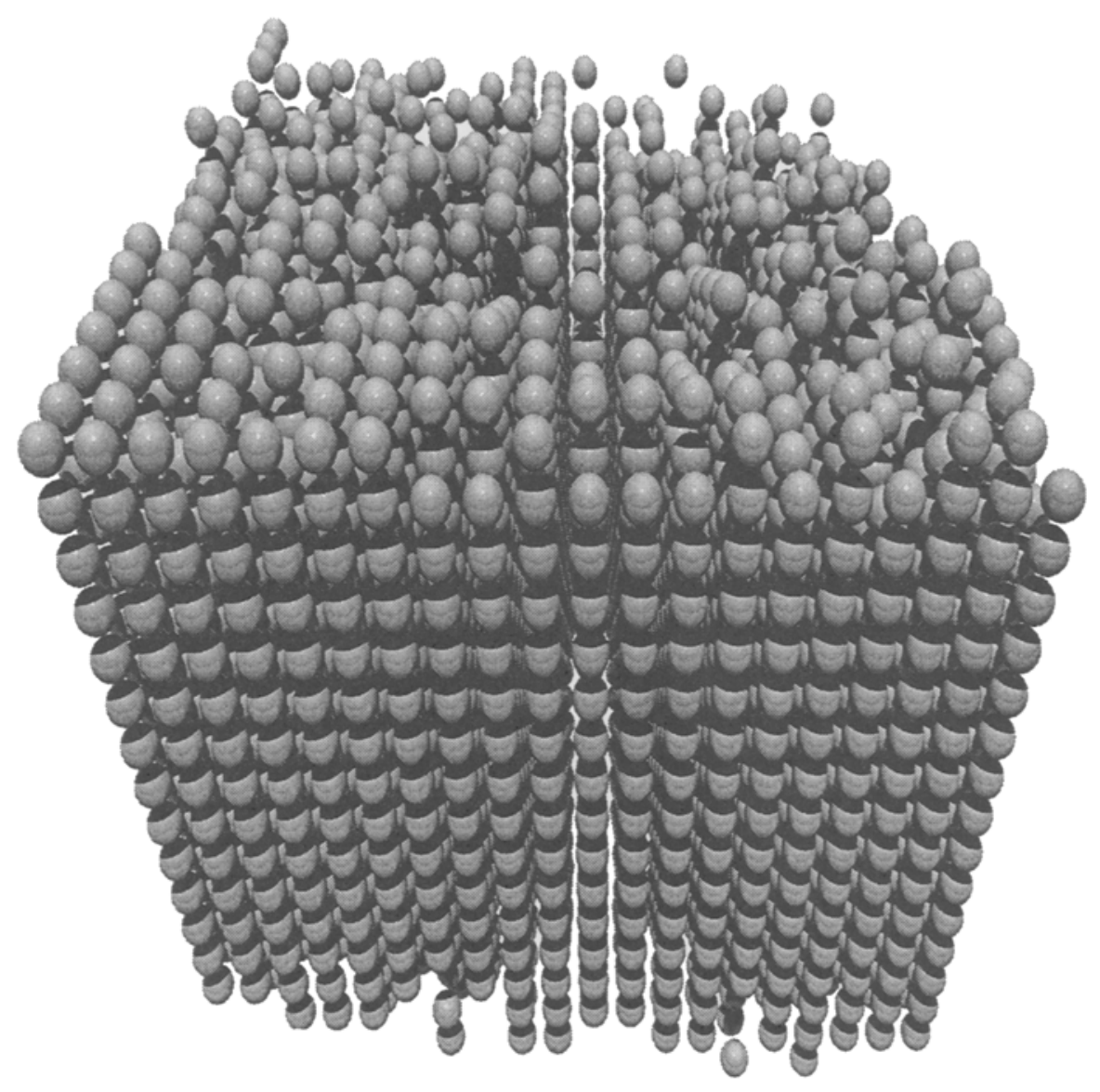

Fig. 1. A BD-sample

It should be mentioned that the restriction to the lattice $\mathbb{Z}^{n}$ is not a crucial one. One the one hand, the problem is affinely invariant and, on the other hand, the whole model can be rephrased in a purely combinatorial form that relies only on incidences anyway.

Clearly, the best known and most important part of the general area of tomography is computerized tomography, an invaluable tool in medical diagnosis and many other areas including biology, chemistry and material sciences. While the mathematics of computerized tomography is quite well understood and utilized in every day practice, bringing down the resolution to the atomic scale 
changes this inverse problem drastically. Continuous methods do not seem appropriate anymore. In the following we will show, how the discreteness effects the problem.

\section{Uniqueness Results}

Clearly, when real world data is given, it is not enough to check consistency (up to data errors) and reconstruct an object compatible with the given X-ray data. It is absolutely necessary to have some uniqueness and stability results as well. It would not be a great help to be able to reconstruct an object that satisfies all the given X-ray constraints and fits the data perfectly if there were many other solutions of very different shape and nature. So let us begin by dealing with the question of unique determination of finite lattice sets by their $\mathrm{X}$-rays.

In the following let $\mathcal{F}^{n}=\left\{F: F \subset \mathbb{Z}^{n} \wedge F\right.$ is finite $\}$ and $S^{n}=\{\operatorname{lin}\{u\}$ : $\left.u \in \mathbb{E}^{n} \backslash\{0\}\right\}$. The elements of $\mathcal{F}^{n}$ are called lattice sets. Quite typically, we have some additional a priori information available. This is modeled by considering a suitable subset $\mathcal{G}$ of $\mathcal{F}^{n}$. For instance, $\mathcal{G}$ may incorporate some contiguity condition that reflects that the crystalline structures that are to be reconstructed do not consist of 'scattered' atoms but are highly connected. In most cases it will also be necessary to consider subsets $\mathcal{T}$ of $\mathcal{S}^{n}$ since electron microscopic images of high enough resolution can only be obtained in certain directions. An important such subset of $\mathcal{S}^{n}$ is the set, $\mathcal{L}^{n}=\left\{\operatorname{lin}\{u\}: u \in \mathbb{Z}^{n} \backslash\{0\}\right\}$ of lattice lines.

We say that $\mathcal{G}$ is determined by $m X$-rays with respect to $\mathcal{T}$ if and only if there exist $S_{1}, \ldots, S_{m} \in \mathcal{T}$ such that the following holds: When $F_{1}, F_{2} \in \mathcal{G}$ and $X_{S_{j}} F_{1}=X_{S_{j}} F_{2}$ for $j=1, \ldots, m$, then $F_{1}=F_{2}$.

Let us begin with a trivial uniqueness results that indicates already the fundamental difference between discrete and continuous tomography.

\subsection{With respect to $\mathcal{S}^{n}$, the class $\mathcal{F}^{n}$ is determined by one $X$-ray.}

Of course, an X-ray line in a non-lattice direction either misses $\mathbb{Z}^{n}$ or, if it contains a lattice point, this lattice point is the unique lattice point on this line. From a certain point of view, (2.1) solves the problem. However, X-rays in nonlattice directions are not practical at all. The resolution coming from such $\mathrm{X}$-rays would not be good enough, the image would typically be completely blurred. In fact good resolution can only be achieved in practice in certain main directions of the lattice.

Another quite simple uniqueness theorem is due to [26].

2.2 For $n=2$, any $|F|+1 X$-rays in pairwise non-parallel directions determine $F$ within $\mathcal{F}^{n}$.

Some extensions of this result are contained in [5]. The problem with (2.2) in practice is that the typical atomic structures that have to be reconstructed comprise about $10^{6}$ to $10^{9}$ atoms. That means that we would need an extremely 
large number of X-ray images to be certain that the object is uniquely determined. However, after about 3 to 5 images taken by high resolution electron microscopy, the object is destroyed by the energy of the radiation, i.e. the object changes and after just a few X-rays it is no longer the original object that is 'seen' by the subsequent X-rays. In fact, it is easy to see that a fix number of $\mathrm{X}$-rays is not sufficient to determine finite lattice sets uniquely.

In order to obtain positive results there seem to be only two options. The first option is to restrict the class of lattice sets under consideration. This general approach is quite reasonable since there is lots of information from physics waiting to be utilized. In terms of mathematical uniqueness results there is the following theorem of [12] for the class $\mathcal{C}^{n}$ of convex lattice sets, i.e. finite subsets $F$ of $\mathbb{Z}^{n}$ such that $F=\mathbb{Z}^{n} \cap \operatorname{conv}(F)$.

2.3 The class $\mathcal{C}^{n}$ is determined by suitable 4 and any $7 X$-rays in pairwise non-parallel coplanar lattice directions.

It is an open problem where the later result persists when the assumption of coplanarity of the lattice directions is abandoned. In practice this restriction is satisfied since for technical reasons the 'tilting' of the microscope is confined to rotations about an axis, hence all $\mathrm{X}$-ray directions lie in a common plane. This means, further, that the reconstruction can be done slice by slice, hence the underlying problem is essentially 2-dimensional. Let us point out that among the 'good' sets of directions in terms of $(2.3)$ there are many that do have components of quite small absolute value which can be handled in practice. Examples for such sets in $\mathbb{E}^{2}$ are $\{(1,0),(1,1),(1,2),(1,5)\},\{(1,0),(2,1),(0,1),(-1,2)\}$ and $\{(2,1),(3,2),(1,1),(2,3)\}$, see [12] and also [3]. While this result is quite reassuring, it is only practical in a very restricted setting. There may be some applications, for instance in colloid physics, but the main demand for mathematical methods for solving the inverse problems of discrete tomography comes from applications that involve the reconstruction of highly non convex objects. In particular, quality control in certain stages of chip production involves the detection of 'bumps' on the surface of silicon chips, hence convexity is not an appropriate condition in this situation.

It may be possible to weaken the assumptions of convexity so that only convexity in the X-ray directions together with strong connectivity assumptions are needed. At present, however, the above result may be mathematically satisfactory, in practice it is largely irrelevant.

So far, we have considered the problem of unique determination under the assumption, that we choose the directions in which X-rays are taken beforehand. A reasonable approach may, on the other hand, be to take the first $\mathrm{X}$-ray in an arbitrary direction but then use the information gained from analyzing the image in order to determine the next direction in which an X-ray is taken. For the third direction, one could then use the complete information given by the first two X-rays and so on. This approach of successive determination leads to strong uniqueness results even for higher dimensional X-rays and even for more general sets then lattice sets, see [12]. In particular 


\section{4 $\mathcal{F}^{n}$ can be successively determined by $2 X$-rays.}

Again, while seeming to be satisfactory, this result is not practical at all. The reason is that the second X-ray has to be so 'skew' that one cannot produce images of high enough resolution in this direction.

Except for some results in more restricted situations, these are the only uniqueness results available at present. So what can be done?

Of course, the first question would be whether we have used all the crystallographic knowledge that there is. While this is certainly not the case is has not been possibly yet to identify reasonable mathematical constraints based on this crystallographic knowledge that reduce the relevant classes of lattice sets enough so as to lead to uniqueness theorems. In fact, there are examples showing that no fixed number of lattice X-rays suffices for determination that look like a solid crystalline blocks with 'just a few impurities.'

In view of the lack of satisfactory uniqueness theorems it seems that at least for the time being we have to settle for less. We may be satisfied by determining the 'core' of all solutions, the set of all invariant points that must belong to all solutions or at least to most in a sense that has to be made precise. We might also be satisfied by determining a 'typical' solution. We will come back to these aspects later. Another practically quite satisfying option could be to check uniqueness algorithmically. While we do not have general a priori uniqueness guarantees it is certainly true that in many practical applications sets to be reconstructed are determined uniquely by the available information. So, an efficient procedure to check uniqueness algorithmically might actually be all that is needed in practice. This brings up the algorithmic aspect of discrete tomography in the context of uniqueness. But it is certainly clear that efficient procedures for reconstruction are needed anyway. We will study algorithmic questions in the next sections.

\section{Computational Complexity}

In this section we state results dealing with the computational complexity of the questions of checking consistency of X-ray data, of determining uniqueness of given solutions and, of course, of finally reconstructing the objects. In the following we will focus on the full family $\mathcal{F}^{n}$, and the results will only be stated for that case. However, most of the results hold for a great variety of other subclasses $\mathcal{G}$ as well, without any significant change, see [14].

Suppose that $S_{1}, \ldots, S_{m} \in \mathcal{L}^{n}$ are $m \geq 2$ lines specified beforehand. In the inverse problem RECONSTRUCTION $\left(S_{1}, \ldots, S_{m}\right)$, we are given candidate functions

$$
f_{i}: \mathcal{A}\left(S_{i}\right) \rightarrow \mathbb{N}_{0}, \quad i=1, \ldots, m
$$

with finite support and want to find a set $F \subset \mathbb{Z}^{n}$ with corresponding X-rays or decide that no such $F$ exists. Since for the purpose of computational complexity theory decision problems are more appropriate than reconstruction problems, we consider also the problem Consistency $\left(S_{1}, \ldots, S_{m}\right)$ whose instances are just 
the same but whose task is restricted to the decision whether a solution exists (without being obliged to produce one). Similarly, UNIQUENEsS $\left(S_{1}, \ldots, S_{m}\right)$ asks whether, given a solution $F$, there exists another one. (Asking the question in this way puts the problem into the class $\mathbb{P}$.) Clearly, when investigating the computational complexity of such problems in the usual binary Turing machine model one has to describe suitable finite data structures and specify the problems accordingly. We refrain from doing this here but refer the reader to [14].

Here is a tractability results.

3.1 Whenever $S_{1}, S_{2} \in \mathcal{L}^{n}$, all three problems, CONSISTENCY $\left(S_{1}, S_{2}\right)$, UNIQUENESS $\left(S_{1}, S_{2}\right)$ and RECONSTRUCTION $\left(S_{1}, S_{2}\right)$ can be solved in polynomial time.

Proofs for the planar case can be found in [7], [16], [27], [28] or [2]. A particularly interesting way of looking at it is to interpret the problem as 2-PARTITIONMATROID-INTERSECTION. While this interpretation might not lead to the most efficient algorithm, it provides an elegant way of generalizing the results to higher dimensional $\mathrm{X}$-rays and arbitrary dimensions. In fact, arbitrary linear optimization problems over the intersection of two matroids can be solved in polynomial time, see [8], [9]. So the case of just two X-rays is algorithmically tractable, see [13].

However, two $\mathrm{X}$-rays usually do not determine finite lattice sets uniquely; hence, we would like to extend these tractability results to a greater number of X-rays. Unfortunately, there is a drastic jump in complexity from $m=2$ to $m=3$.

3.2 For $n \geq 2$ and $m \geq 3$ different lines $S_{1}, \ldots, S_{m}$ in $\mathcal{L}^{n}$, the problems $\operatorname{CONSISTENCY}\left(S_{1}, \ldots, S_{m}\right)$ and UNIQUENESS $\left(S_{1}, \ldots, S_{m}\right)$ are $\mathbb{N}$-complete in the strong sense; RECONSTRUCTION $\left(S_{1}, \ldots, S_{m}\right)$ is $\mathbb{N P}$-hard.

This intractability result due to $[14]$ generalizes and sharpens previously known results. N. Young (private communication) showed the $\mathbb{N}$-hardness of Consistency $\left(S_{1}, S_{2}, S_{3}, S_{4}\right)$ when $S_{1}, \ldots, S_{4}$ are the four coordinate axes in $\mathrm{E}^{4}$. He used this result to obtain the NIP-hardness of a consistency problem for $n=2$ and $m=4$; however, the fourth direction is part of the input, so this is much weaker than the corresponding case of (3.2). Also, it is shown in [21, Section 4.1] (in the context of contingency tables), by a transformation from LATIN-SQUARE, that Consistency $\left(S_{1}, S_{2}, S_{3}\right)$ is $\mathbb{N P}$-complete when $S_{1}, S_{2}, S_{3}$ are the coordinate axes in $\mathbb{E}^{3}$.

The hardness results of [14] are given by transformations from the well known $\mathbb{N P}$-complete problem 1-IN-3-SAT. The constructions shows that hardness does occur in situations which are not too far off from practice. In fact, the constructed objects are again solid crystals with just a few impurities and can represent, in principle, physically reasonable objects. Therefore (3.2) seems to be an appropriate explanation of the algorithmic difficulties observed in practice. An extension of these hardness results is given in [19].

There are some other related complexity results, particularly those of [4] and [31] for polyominoes in the plane. 


\section{Algorithmic Approaches in the Presence of $\mathbb{N P}$-Hardness}

Even though our problems are $\mathbb{N} \mathbb{P}$-hard, they have to be solved in practice. Various approaches have been suggested for dealing with Consistency $\left(S_{1}, \ldots, S_{m}\right)$, Uniqueness $\left(S_{1}, \ldots, S_{m}\right)$ and Reconstruction $\left(S_{1}, \ldots, S_{m}\right)$ in practice. See [19] for an account of the success and failure of many of those techniques. For simplicity, we restrict the following exposition to the problem of checking data consistency, and we will always assume that $S_{1}, \ldots, S_{m}$ are $m \geq 2$ different lattice lines.

It is easy to see, that Consistency $\left(S_{1}, \ldots, S_{m}\right)$ can be formulated as an integer linear programming problem whose variables correspond to the possible positions of elements of a solution. In fact, the grid $G$ associated with a given instance of the problem consists of all (finitely many) lattice points that arise as points of intersection of $m$ lines parallel to $S_{1}, \ldots, S_{m}$, respectively, whose candidate function value is nonzero, i.e.

$$
G=\mathbb{Z}^{n} \cap \bigcap_{i=1}^{m} \bigcup_{T \in \mathcal{T}_{i}} T
$$

where $\mathcal{T}_{1}, \ldots, \mathcal{T}_{m}$ denote the supports of the given candidate functions $f_{1}, \ldots, f_{m}$, respectively. The incidences of $G$ and $\mathcal{T}_{i}$ can be encoded by an incidence matrix $A_{i}$. If $G$ consist of, say, $N$ points, $M_{i}=\left|\mathcal{T}_{i}\right|$ for $i=1, \ldots, m$, and $M=M_{1}+$ $\ldots+M_{m}$, then the incidence matrices $A_{i}$ are in $\{0,1\}^{M_{i} \times N}$, and can be joined together to form a matrix $A \in\{0,1\}^{M \times N}$. Identifying a subset of $G$ with its characteristic vector $x \in\{0,1\}^{N}$, the reconstruction problem amounts to solving the integer linear feasibility program

$$
A x=b \text {, s.t. } x \in\{0,1\}^{N},
$$

where $b^{T}=\left(b_{1}^{T}, \ldots, b_{m}^{T}\right)$ contains the corresponding values of the candidate functions $f_{1}, \ldots, f_{m}$ as the right hand sides of $A_{1}, \ldots, A_{m}$, respectively.

Since linear programming problems can be solved in polynomial time the first natural approach is to consider the $L P$-relaxation

$$
A x=b \wedge 0 \leq x \leq 1,
$$

of (1), where, as usual, ' $\leq$ ' is to be understood componentwise; see [11]. Since linear programming codes are available for solving these problems very efficiently for all sizes of crystalline structures that are relevant in practice, computation time is not much of an issue for this heuristic. However, the solution is usually far from being integer and it does not seem completely justified to interpret fractional components of solutions as probabilities that the corresponding points belong to a (typical) solution.

In an improvement strategy $N$. Young studied the effect of such an interpretation followed by a subsequent randomized rounding. Here an atom is placed at the corresponding lattice point with the probability coming from the fractional 
solution produced by the LP-solver. This way an approximative solution to the consistency problem is produced. Compared to other heuristics for the problems in discrete tomography, known bounds for such solutions are, however, in general rather weak. We will give some bound on approximation errors for various heuristics in Section 5.

Of course, one cannot expect too tight a priori error bounds for polynomialtime approximative heuristics in general. But again, for all practical purposes it is nice but not absolutely necessary to have such a priori bounds. In fact, a method that would, in the course of the run of the algorithm, provide upper and lower bounds might be all that is needed. One can then run the algorithm on the given data until the gap between upper and lower bounds is small enough and then terminate with an approximate solution including a performance guaranty. The next section will give the basic idea of such an algorithm, the branch-and-cut method.

\section{Polytopes in Discrete Tomography}

The idea behind the branch-and-cut method is to try to approximate the polyhedral structure of the convex hull

$$
P(A, b)=\operatorname{conv}\left\{x \in\{0,1\}^{N}: A x=b\right\},
$$

of all solutions of (1). In the algorithm, we begin with an LP-relaxation of the integer programming problem (1), compute a solution, and check whether it is already in $\{0,1\}^{N}$. If this is the case, we stop with a consistent solution for the given instance. Otherwise we try to find a (facet-defining) constraint for $P(A, b)$ that is violated by the LP solution. This means, we try to find a cutting plane that is in a sense best possible for providing a deep cut. If such a separating hyperplane can be found, we add the corresponding constraint to the current LP and repeat the procedure. It may actually happen that the LP-solution found is not in $\{0,1\}^{N}$ but we are still not able to produce a separating hyperplane. In that case, we use a branch-and-bound paradigm and proceed in the same algorithmic framework as before - just splitting the problem into subproblems. Such an approach has been successfully utilized for many problems in combinatorial optimization, most notably for the traveling salesman problem, see [20], [25] for surveys on the polyhedral theory and on polyhedral computations for the traveling salesman problem, and see [6] for a geometric introduction into the general concept underlying polyhedral combinatorics.

Since, in general, computing the dimension of $P(A, b)$ is $\mathbb{N P}$-hard by $(3.2)$ and since it does not seem wise to try to 'jump to a solution' in one step anyway, $P(A, b)$ is replaced in the polyhedral study $[17]$ by

$$
T(A, b)=\operatorname{conv}\left\{x \in\{0,1\}^{N}: A x \leq b\right\} .
$$

Then, of course, we have to model that we are only interested in a solution of maximum cardinality in order to obtain an equivalent problem. This can 
be done by adding the objective to maximize the funktion $x \mapsto e^{T} x$, where $e=(1, \ldots, 1)^{T} \in \mathbb{E}^{N}$.

The tomography polytopes $T(A, b)$ are quite special. Clearly, all tomography polytopes are subpolytopes of the standard cube $\{0,1\}^{N}$ whose coefficient matrix $A$ is a 0-1-matrix with a particular structure. Specifically, all submatrixes corresponding to just two directions are totally unimodular - another explanation why the case of two directions is simple. It follows from [24] that the combinatorial diameter of a tomography polytope is at most $N$. This means that, in principle, an edge-path could be found leading from 0 to a solution of the problem that is rather short. The main problem of course is, that it is not known how to actually choose the pivot element at each step in order to find such short paths. Of course the underlying physics induces additional structure that can be utilized in the study of the polytopes.

Further, using all sorts of preprocessing techniques, the practically relevant dimensions of these polytopes can be reduced to about $10^{4}$. Judged on the base of the sizes of successfully solved instances of the traveling salesman problem this seems encouragingly small. While the traveling salesman polytopes correspond to the complete graph on the given number of 'cities' and are hence universal, tomography polytopes depend, on the other hand, on the right-hand side $b$. So the most important goal of polytopal investigations is to find large systems of valid inequalities that are facet-defining under week conditions on the right-hand side. In [17] various classes of facet-defining inequalities are determined under very weak assumptions on $b$. Usually it is only necessary to require that all components of $b$ are at least 2 or 3 and that no X-ray line is completely filled with atoms. Using these branch-and-cut techniques one can reconstruct moderately big crystalline structures already. The example of Figure 1 was produced by a (not yet fully developed) algorithm of this kind. It shows a 3D-phantom - whose $\mathrm{X}$-ray data in directions $(0,1,0),(1,4,0)$ and $(-1,4,0)$ were given to us by $\mathrm{P}$. Schwander - that turns out to be uniquely determined by the X-rays in these directions. (One should regard the front plane as the $x y$-plane while the different $z$-coordinates correspond to planes parallel to the $x y$-plane).

\section{Approximation Algorithms with A Priori Performance Guarantees}

In the following we study some simple heuristics that can be fully analyzed and give approximation guarantees that yield a constant relative error. It is clear that these techniques by themselves will not solve the problem since their performance in terms of approximating a solution is still rather poor. However, it is first of all reassuring that simple techniques yield such a small relative error, and on the other hand such techniques can be used as a preprocessing step in order to find good bounds in any branch-and-bound based approach.

The two problems that we are going to consider now are BEST-INNERFIT $\left(S_{1}, \ldots, S_{m}\right)$ [BIF] and BEST-OUTER-FIT $\left(S_{1}, \ldots, S_{m}\right)$ [BOF]. Given candidate functions $f_{1}, \ldots, f_{m}$, the tasks of these problems are to find a set $F \subset G$, 
respectively. For $[\mathrm{BIF}],|F|$ must be maximal such that $X_{S_{i}} F(T) \leq f_{i}(T)$ for all $T \in \mathcal{T}_{i}$ and $i=1, \ldots, m$, while for $[\mathrm{BOF}],|F|$ must be minimal such that $X_{S_{i}} F(T) \geq f_{i}(T)$ for all $T \in \mathcal{T}_{i}$ and $i=1, \ldots, m$.

For an analysis of these and other approximation problems for discrete tomography see [18]. The first strategy for [BIF] that is fully analyzed is that of the greedy heuristic: place points into the grid of the given instance whenever that is possible without violating the constraints. Then one obtains the following bound.

\subsection{If $F$ is a solution of [BIF] and $L$ is a greedy solution then $m|L| \geq|F|$.}

It may be worthwhile to point out that the greedy strategy is very flexible and allows various specifications for breaking the ties between different choices for points to be placed next. For example, the X-ray data can be used in a way that is very similar to back-projection techniques to express preferences. In addition, connectivity of the solution (in a sense that is justified by the physical structure of the analyzed material) can be rewarded. Similarly, information of neighboring layers can be taken into account in a layer-wise reconstruction of a 3D-object.

While the above result follows from work of [23], the next bound is due to [18]. It uses the notion of an (increasing) $r$-change: $r$ points of a feasible set $F G$ are deleted and $r+1$ points of $G \backslash F$ are inserted instead without destroying the feasibility. Clearly, $r$-changes are more powerful than just greedy insertion (which actually can be regarded as a 0-change strategy).

6.2 Let $t \in \mathbb{N}$, let $F$ be a solution of [BIF] and let $L \subset G$ such that no further $r$-change is possible then

$$
|L| \geq\left(\frac{2}{m}-\epsilon(t)\right)|F|, \text { where } \epsilon(t) \rightarrow 0 \text { for } t \rightarrow \infty
$$

Similar results car be obtained for [BOF] see [18]. Rather than specifying the algorithms precisely - they are based again on greedy strategies, (decreasing) $r$ changes and matching techniques - let us just state a result for [BOF] that corresponds to $(6.2)$.

6.3 For every $\epsilon>0$ there is a polynomial-time algorithm that, given an instance of [BOF] produces a set $L$ that is feasible and whose cardinality exceeds that of an optimal solution by a factor at most

$$
\frac{2}{3}+\frac{1}{2}+\ldots+\frac{1}{m}+\epsilon
$$

Another simple but useful observation is that if we compute a basic optimal solution of the LP-relaxation $N-M$ of the 0 -1-constraints of (1) are already satisfied. 


\section{$7 \quad$ Dealing with Nonuniqueness}

So far, we have mainly studied the question of checking data consistency. A problem of similar (or even greater) importance, is that of dealing with nonuniqueness. Again, if the data given in practice allow many solutions that are extremely different, the relevance of finding one of them is not apparent any more. There are various suggestions for how to deal with the problem of nonuniqueness.

If we use the fact, that in the practical application for material sciences that motivated this study the directions in which the X-ray images are taken are coplanar, then the objects can be reconstructed layer by layer. Suppose that the first layer has been reconstructed and that it is uniquely determined by the given information (or known beforehand). Then it may be reasonable to assume, that the second layer does not vary too much from the first. We can easily model that by adding an objective function to our reconstruction problem for the second layer. In fact the incidence vector of the solution for the first layer provides a linear objective function whose maximization leads to a solution for the second layer that is in a certain sense closest to that for the first one. In case of just two X-rays such an approach was suggested by [16].

[11] uses an interior point method for solving the LP-relaxation

$$
\max e^{T} x \text { s.t. } A x \leq b \wedge 0 \leq x \leq 1
$$

of the (monotone vorsion of the) reconstruction problem in order to identify positions that are uniquely determined by the given data. In fact such an approach produces a point that is interior to the optimal face of the LP-polytope. So if a component of the LP-solution is 0 or 1 then the same is true for all solutions and hence for all 0-1 solutions. While by [14] the problem of detecting whether a given subset of $G$ belongs to all solutions is again $\mathbb{N P}$-hard (see also [19]), it is demonstrated in [11] that for certain phantoms such an approach produces quite a large number of fixed variables. Note that the problem of deciding whether a subset of a possible solution can actually be extended to a full solution is again $\mathbb{N P}$-complete, see [14]. It should be noted that the notion of additivity as introduced by [10] and extended by [1] is actually equivalent to 'substructure uniqueness' for the LP-relaxation.

All of the above mentioned techniques can be used and combined in practice to reduce nonuniqueness but it will certainly still be mandatory to take additional physical constraints into account in order to be able to produce solutions that resemble the actual physical objects.

\section{Imprecise Data}

Even if we manage to reduce nonuniqueness to a large extent and solve the reconstruction problem efficiently in practice, there is still the problem of imprecise data. Of course there is always some noise involved when physical measurements are taken but for the problem under consideration also some systematic error is present. In fact, the sample that is to be studied has to be prepared for electron 
microscopy by thinning it out so as to let the electron waves penetrate it. For technical reasons this is done in such a way that the object is much thinner in the middle than at the boundary where it is attached to some position control element. This means that an X-ray taken in some direction 'sees' the crystalline structure in the middle of the object but is absorbed towards the boundary. Taking a second direction, one has the same effect, just that the parts of absorption at the boundary do not coincide. That means that in practice the data that one gets from the measurements actually correspond to slightly different objects.

There are various ways of modeling data inconsistency and error. General techniques for dealing with ill-posed problems can be applied by using an objective function that minimizes the distance of the computed right-hand side for a found solution from the given right-hand side data. This can be done in various different ways. A promising suggestion of this kind is due to [30] since it allows to interpret solutions as best-approximations in the sense of maximum likelihood. The method is, however, again based on the LP-relaxation of the underlying 0-1-programming problem. It will be particularly intriguing to find out on the basis of real-world data to what extent 'severe artifacts' are introduced by methods based on the underlying LP-relaxation. It is certainly clear that LP-based techniques are generally not capable of capturing the discreteness of the problem. Maximum likelihood strategies on all possible $0-1$ solutions are on the other hand algorithmically quite demanding.

\section{$9 \quad$ Final Remarks}

The problems of discrete tomography are practically important, mathematically rich (involving methods from areas like combinatorial optimization, number theory, geometry, combinatorics, stochastics, etc.) and algorithmically challenging. During the last few years there has been considerable progress in attacking these problems. However, there is still a lot of work to be done to finally create a tool that is as developed and satisfactory for the application in material sciences as is computer tomography in its medical and other applications.

\section{References}

1. R. Aharoni, G.T. Herman, and A. Kuba, Binary vectors partially determined by linear equation systems, Discrete Math. 171 (1997), 1-16.

2. R.P. Anstee, The network flow opproach for matrices with given row and column sums, Discrete Math. 44 (1983), 125-138.

3. E. Barcucci, A. Del Lungo, M. Nivat, and R. Pinzani, X-rays choracterizing some classes of digital pictures, preprint.

4. - Reconstructing convex polyominoes from their horizontal and vertical projections, Th. Comput. Sci. 155 (1996), 321-347.

5. G. Bianchi and M. Longinetti, Reconstructing plane sets from projections, Discrete Comput. Geom. 5 (1990), 223-242.

6. T. Burger and P. Gritzmann, Polytopes in combinatorial optimization, Geometry at Work (C. Gorini, ed.), Math. Assoc. Amer., 1997, in print. 
7. S.K. Chang, The reconstruction of binary patterns from their projections, Comm. ACM 14 (1971), no. 1, 21-25.

8. J. Edmonds, Maximum matching and a poiydedron with 0,1-vertices, J. Research National Bureau of Standards 69B (1965), no. 1 and 2, 125-130.

9. - Matroid intersection, Ann. Discrete Math. 4 (1979), 39-49.

10. P.C. Fishburn, J.C. Lagarias, J.A. Reeds, and L.A. Shepp, Sets uniquely determined by projections on axes. II. discrete case, Discrete Math. 91 (1991), 149-159.

11. P.C. Fishburn, P. Schwander, L.A. Shepp, and J. Vanderbei, The discrete Radon transform and its approximate inversion via linear programming, Discrete Appl. Math. 75 (1997), 39-62.

12. R.J. Gardner and P. Gritzmann, Discrete tomography: Determination of finite sets by $X$-rays, Trans. Amer. Math. Soc 349 (1997), 2271-2295.

13. R.J. Gardner, P. Gritzmann, and D. Prangenberg, On the reconstruction of binary images from their discrete Radon transforms, Proc. Intern. Symp. Optical Science, Engineering, and Instrumentation (Bellingham, WA), SPIE, 1996, pp. 121-132.

14. - Computational complexity issues in discrete tomography: On the reconstruction of finite lattice sets from their line $X$-rays, submitted, 1997.

15. - On the computational complexity of determining polyatomic structures by $X$-rays, submitted, 1997.

16. J.J. Gerbrands and C.H. Slump, A network flow approach to reconstruction of the left ventricle from two projections, Computer Graphics and Image Processing 18 (1982), 18-36.

17. P. Gritzmann and S. de Vries, Polytopes for discrete tomography, in preparation.

18. P. Gritzmann, S. de Vries, and M. Wiegelmann, On the approximate reconstruction of binary images from their discrete X-rays, in preparation, 1997.

19. P. Gritzmann, D. Prangenberg, S. de Vries, and M. Wiegelmann, Success and faiture of certain reconstruction and uniqueness algorithms in discrete tomography, submitted, 1997.

20. M. Grötschel and M. Padberg, Polyhedral theory, The Traveling Salesman Problem (E.L. Lawler, J.K. Lenstra, A.H.G. Rinnoy Kan, and D.B. Shmoys, eds.), WileyInterscience, Chichester, 1985, pp. 251-305.

21. R.W. Irving and M.R. Jerrum, Three-dimensional statistical data security problems, SIAM J. Comput. 23 (1994), 170-184.

22. C. Kisielowski, P. Schwander, F.H. Baumann, M. Seibt, Y. Kim, and A. Ourmazd, An approach to quantitative high-resolution transmission electron microscopy of crystalline materials, Ultramicroscopy 58 (1995), 131-155.

23. B. Korte and D. Hausmann, An analysis of the greedy heuristic for independence systems, Ann. Discrete Math. 2 (1978), 65-74.

24. D. Naddef, The Hirsch conjecture is true for $(0,1)$-polytopes, Math. Prog. 45 (1989), 109-110.

25. M. Padberg and M. Grötschel, Polyhedral computations, The Traveling Salesman Problem (E.L. Lawler, J.K. Lenstra, A.H.G. Rinnoy Kan, and D.B. Shmoys, eds.), Wiley-Interscience, Chichester, 1985, pp. 307-360.

26. A. Rényi, On projections of probability distributions, Acta Math. Acad. Sci. Hungar. 3 (1952), 131-142.

27. H.J. Ryser, Combinatorial properties of matrices of zeros and ones, Canad. J. Math. 9 (1957), 371-377.

28. ___ Combinatorial mathematics, Mathem. Assoc. Amer. and Quinn \& Boden, Rahway, NJ, 1963. 
29. P. Schwander, C. Kisielowski, M. Seibt, F.H. Baumann, Y. Kim, and A. Ourmazd, Mapping projected potential, interfacial roughness, and composition in general crystalline solids by quantitative transmission electron microscopy, Physical Review Letters 71 (1993), 4150-4153.

30. Y. Vardi and D. Lee, The discrete Radon transform and its approximate inversion via the ELM algorithm, preprint, 1997.

31. G.J. Woeginger, The reconstruction of polyominoes from their orthogonal projections, preprint. 